\title{
NUCLEUS-NUCLEUS COLLISIONS
}

PROCEEDINGS OF THE CONFERENCE: BOLOGNA 2000 STRUCTURE OF THE NUCLEUS AT THE DAWN OF THE CENTURY 


\section{THE SCIENCE AND CULTURE SERIES - ADVANCED SCIENTIFIC CULTURE}

Series Editor: A. Zichichi, European Physical Society, Geneva, Switzerland

Series Editorial Board: P. G. Bergmann, J. Collinge, V. Hughes, N. Kurti, T. D. Lee, K. M. B. Siegbahn, G. 't Hooft, P. Toubert, E. Velikhov,

G. Veneziano, G. Zhou

1. Nucleus-Nucleus Collisions

Bologna 2000. Structure of the Nucleus at the Dawn of the Century

2. Nuclear Structure

Bologna 2000. Structure of the Nucleus at the Dawn of the Century

3. Hadrons, Nuclei and Applications

Bologna 2000. Structure of the Nucleus at the Dawn of the Century 


\title{
NUCLEUS-NUCLEUS COLLISIONS
}

PROCEEDINGS OF THE CONFERENCE: BOLOGNA 2000 STRUCTURE OF THE NUCLEUS AT THE DAWN OF THE CENTURY

\author{
Bologna, Italy 29 May - 3 June 2000
}

Editors

\section{Giovanni C. Bonsignori Mauro Bruno}

Dipartimento di Fisica dell'Università di Bologna and INFN-Sezione di Bologna, Italy

\section{Alberto Ventura}

Ente Nuove Tecnologie, Energia e Ambiente and INFN Bologna, Italy

\section{Dario Vretenar}

Physics Department, University of Zagreb, Croatia

Series Editor

A. Zichichi 


\section{Published by}

World Scientific Publishing Co. Pte. Ltd.

P O Box 128, Farrer Road, Singapore 912805

USA office: Suite 1B, 1060 Main Street, River Edge, NJ 07661

UK office: 57 Shelton Street, Covent Garden, London WC2H 9HE

\section{British Library Cataloguing-in-Publication Data}

A catalogue record for this book is available from the British Library.

\section{NUCLEUS-NUCLEUS COLLISIONS}

Proceedings of the Conference: Bologna 2000

Structure of the Nucleus at the Dawn of the Century

Copyright $(2001$ by World Scientific Publishing Co. Pte. Ltd.

All rights reserved. This book, or parts thereof, may not be reproduced in any form or by any means, electronic or mechanical, including photocopying, recording or any information storage and retrieval system now known or to be invented, without written permission from the Publisher.

For photocopying of material in this volume, please pay a copying fee through the Copyright Clearance Center, Inc., 222 Rosewood Drive, Danvers, MA 01923, USA. In this case permission to photocopy is not required from the publisher.

Printed in Singapore. 


\section{CONTENTS}

Foreword

The Editors

Welcome Address xix

F. Roversi-Monaco

Committees and Sponsors $\mathrm{xxi}$

Photographs xxiii

\section{Opening Lecture}

Antimatter - Past, Present and Future

A. Zichichi

\section{Introductory Talk}

What Can Nuclear Collisions Teach Us about the Boiling

of Water or the Formation of Multi-Star Systems?

D. H. E. Gross

Section I. Quark and Gluon-Plasma Phase Transition and Relativistic Heavy-Ion Reactions

Results from $\mathrm{Pb}-\mathrm{Pb}$ Collisions at CERN SPS

L. Riccati and E. Scomparin

The Search for the QGP: A Critical Appraisal

H. Satz

Strange Baryon Signals of a New State of Matter in Lead-Lead Collisions at the CERN SPS

F. Antinori for the WA97 Collaboration 
Cooper-Mesons in the Color-Flavor-Locked Superconducting

Phase of Dense QCD
A. Wirzba

Charmonium Suppression in $\mathrm{Pb}-\mathrm{Pb}$ Collisions and

Quark-Gluon Deconfinement

A. B. Kurepin for the NA50 Collaboration

Particle Production in $\mathrm{Pb}+\mathrm{Pb}$ Collisions at $158 \mathrm{GeV} / \mathrm{Nucleon}$

in the NA49 Detector

$H$. Ströbele for the NA49 Collaboration

Exploring the Chiral Phase Transition in High-Energy Collisions

J. Randrup

Nuclear Collective Flow in Heavy Ion Collisions at SIS Energies

N. Bastid for the FOPI Collaboration

Hadron Observables from Hadronic Transport Model with

Jet Production at RHIC

Y. Nara

Simultaneous Heavy Ion Dissociation at Ultrarelativistic Energies

I. A. Pshenichnov, J. P. Bondorf, S. Masetti,

I. N. Mishustin and A. Ventura

Direct Photon Production in $158 \mathrm{~A} \mathrm{GeV}{ }^{208} \mathrm{~Pb}+{ }^{208} \mathrm{~Pb}$ Collisions

T. Peitzmann for the WA98 Collaboration

Deformation and Orientation Effects in Uranium-on-Uranium

Collisions at Relativistic Energies

Bao-An $\mathrm{Li}$

Subthreshold Heavy-Meson and Antiproton Production in the

Nucleus-Nucleus Collisions

A. T. D'Yachenko

Pion Imaging at the AGS

S. Y. Panitkin for the E895 Collaboration 
Directed Flow in $4.2 \mathrm{~A} \mathrm{GeV} / \mathrm{C} \mathrm{C}+\mathrm{C}$ and $\mathrm{C}+\mathrm{Ta}$ Collisions

Fragmentation of Very High Energy Heavy Ions

M. Giorgini and S. Manzoor

Section II. Liquid-Gas Phase Transitions in Nuclear Matter

Phase Transition in Finite Systems

Ph. Chomaz, V. Duflot and F. Gulminelli

Present Status and Future Prospects of Investigations of the Liquid-Gas Phase Transition C.-K. Gelbke

Critical Phenomena in Finite Systems

A. Bonasera, T. Maruyama and S. Chiba

Experimental Signals of the First Phase Transition of Nuclear Matter

B. Borderie

Nuclear Fragmentation, Phase Transitions and their

Characterization in Finite Systems of Interacting Particles

J. M. Carmona, N. Michel, J. Richert and P. Wagner

Topology and Phase Transitions: Towards a Proper Mathematical Definition of Finite $\mathrm{N}$ Transitions

M. Pettini, R. Franzosi and L. Spinelli

Introduction to the Phase Transition Discussion

Ph. Chomaz

Finite Nuclear Fragmenting Systems: An Experimental Evidence of a First Order Liquid-Gas Phase Transition

M. D'Agostino, F. Gulminelli, Ph. Chomaz, M. Bruno,

F. Cannata, N. Le Neindre, R. Bougault, M. L. Fiandri,

E. Fuschini, F. Gramegna, I. Iori, G. V. Margagliotti,

A. Moroni, G. Vannini and E. Verondini 
Phase Transition in XE+SN Central Events between

32 and $50 \mathrm{~A} \mathrm{MeV}$

N. Le Neindre, R. Bougault, Ph. Chomaz,

F. Gulminelli and the INDRA Collaboration

Nuclear Caloric Curve: Influence of the Secondary Decays

227

on the Isotopic Thermometers

A. H. Raduta and A. R. Raduta

Phase Transition Signals in Thermally Excited Nuclei

231

V. E. Viola for the E900 Collaboration

Hydrogen Cluster Multifragmentation and Percolation Models

F. Gobet, B. Farizon, M. Farizon, M. J. Gaillard,

J. P. Buchet, M. Carré, P. Scheier and T. D. Märk

Single Quasiparticle Entropy in Excited Nuclei with $T<1 \mathrm{MeV}$

243

M. Guttormsen, M. Hjorth-Jensen, E. Melby,

J. Rekstad, A. Schiller and S. Siem

Section III. Nuclear Caloric Curve and Thermodynamics of Heavy Ion Collisions

Surveying Temperature and Density Measurements in

Nuclear Calorimetry

G. Raciti for the ALADIN Collaboration

Caloric Curve of Fragmenting Systems

257

C. O. Dorso and A. Chernomoretz

Thermodynamics of Explosions

263

G. Neergaard, J. P. Bondorf and I. N. Mishustin

Microcanonical Investigation of the Recent Nuclear Caloric

269

Curve Experimental Evaluations

A. H. Raduta and A. R. Raduta

Can We Determine the Nuclear Equation of State from Heavy Ion Collisions?

T. Gaitanos, H. H. Wolter, C. Fuchs and A. Faessler 
Thermodynamical Description of Heavy Ion Collisions

T. Gaitanos, H. H. Wolter and C. Fuchs

\section{Section IV. Statistical and Dynamical Aspects of Fragmentation}

Exact Solution of the Statistical Multifragmentation Model 285 and the Liquid-Gas Mixed Phase

K. A. Bugaev, M. I Gorenstein, I. N. Mishustin and

W. Greiner

What Can We Learn from Nuclear Matter Instabilities?

V. Baran, M. Colonna, M. Di Toro,

M. Zielinska-Pfabe and $H$. H. Wolter

Energetic Proton Emission and Reaction Dynamics in Heavy

Ion Reactions Close to the Fermi Energy

R. Coniglione, P. Sapienza, E. Migneco, C. Agodi,

R. Alba, G. Bellia, M. Colonna, A. Del Zoppo,

P. Finocchiaro, V. Greco, K. Loukachine, C. Maiolino,

P. Piattelli, D. Santonocito, P. G. Ventura, N. Colonna,

M. Bruno, M. D'Agostino, M. L. Fiandri, G. Vannini,

P. F. Mastinu, F. Gramegna, I. Iori, L. Fabbietti,

A. Moroni, G. V. Margagliotti, P. M. Milazzo, R. Rui,

F. Tonetto, Y. Blumenfeld and J. A. Scarpaci

New Results on Preequilibrium $\gamma$-Ray Emission and GDR

Saturation on Reactions at $25 \mathrm{~A} \mathrm{MeV}$

G. Cardella, F. Amorini, A. Di Pietro, P. Figuera,

G. Lanzalone, J. Lu, A. Musumarra, M. Papa,

G. Pappalardo, S. Pirrone, F. Rizzo and S. Tudisco

The Onset of Mid-Velocity Emissions in Symmetric Heavy

Ion Reactions

E. Plagnol, J. Lukasik and the INDRA Collaboration

Contribution of Prompt Emissions to the Production of

Intermediate Velocity Light Particles in the ${ }^{36} \mathrm{Ar}+{ }^{58} \mathrm{Ni}$

Reaction at $95 \mathrm{MeV} /$ Nucleon

P. Pawtowski, B. Borderie and the INDRA Collaboration 
Proton Emission Times in Spectator Fragmentation

C. Schwarz for the ALADIN Collaboration

Experimental Evidence for Spinodal Decomposition in

321

Multifragmentation of Heavy Systems

M. F. Rivet for the INDRA Collaboration

Non-Equilibrium Effects on a Second-Order Phase Transition

V. Latora and A. Rapisarda

Multifragmentation of Expanding Nuclear Matter

331

S. Chikazumi, T. Maruyama, K. Niita,

A. Iwamoto and S. Chiba

Contemporary Presence of Dynamical and Statistical Intermediate

Mass Fragment Production Mechanisms in Midperipheral $\mathrm{Ni}+\mathrm{Ni}$

Collisions at $30 \mathrm{MeV} /$ Nucleon

P. M. Milazzo, M. Sisto, G. V. Margagliotti, R. Rui,

G. Vannini, M. Bruno, M. D'Agostino, N. Colonna,

C. Agodi, R. Alba, G. Bellia, M. Colonna, R. Coniglione,

A. Del Zoppo, P. Finocchiaro, C. Maiolino, E. Migneco,

P. Piattelli, D. Santonocito, P. Sapienza, F. Gramegna,

P. F. Mastinu, L. Fabbietti, I. Iori, A. Moroni and

M. Belkacem

External Coulomb and Angular Momentum Influence on

Isotope Composition of Nuclear Fragments

A. S. Botvina

Section V. Intermediate Energy Heavy-Ion Reactions

Isospin Fractionation in Excited Nuclear Systems

S. J. Yennello, M. Veslesky, E. Martin, R. Laforest,

D. Rowland, E. Ramakrishnan, A. Ruangma and

E. M. Winchester

The Disappearance of Flow and the Nuclear Equation of State

G. D. Westfall 
The Backtracing Procedure in Nuclear Physics

P. Désesquelles

$\mathrm{Ni}+\mathrm{Ni}$ Collisions at $32 \mathrm{MeV} / \mathrm{U}$ : Experimental Insight 363 with the INDRA Multidetector

A. M. Maskay, P. Lautesse, P. Désesquelles, E. Gerlic, J. L. Laville and the INDRA Collaboration

Two-Fragment Correlation Function for Quasi-Projectile and Mid-Rapidity Emission

Z. He, R. Roy, L. Gingras, Y. Larochelle, D. Ouerdane,

L. Beaulieu, P. Gagné, X. Qian, C. St-Pierre,

G. C. Ball and D. Horn

Fermion Interferometry in Ni-Induced Reactions at $\mathrm{E} / \mathrm{A}=45 \mathrm{MeV}$

R. Ghetti

Investigation of an Angular Distribution of Protons

in Peripheral and Central Nucleus-Nucleus Collisions at the Momentum of $4.2 \mathrm{~A} \mathrm{GeV} / \mathrm{c}$

M. K. Suleymanov, O. B. Abdinov, Z. Y. Sadigov,

N. S. Angelov, A. C. Vodopyanov and A. A. Kuznetsov

REVERSE Experiment at Laboratori Nazionali del Sud

E. Geraci for the REVERSE Collaboration

REVERSE: The First Experiment with the Chimera Detector

G. Politi for the REVERSE Collaboration

Non Equilibrated IMF Emission in Heavy Ion Collisions around the Fermi Energy

S. Piantelli, L. Bidini, M. Bini, G. Casini, P. R. Maurenzig,

A. Olmi, G. Pasquali, G. Poggi, S. Poggi, A. A. Stefanini and

N. Taccetti

The Complete Fusion and the Competitive Processes in the ${ }^{32} \mathrm{~S}+{ }^{12} \mathrm{C}$ Reaction at $\mathrm{E}\left({ }^{32} \mathrm{~S}\right)=20 \mathrm{MeV} / \mathrm{A}$

S. Pirrone, G. Politi, G. Lanzalone, S. Aiello, N. Arena, Seb. Cavallaro, E. Geraci, F. Porto and S. Sambataro 
A Simple Pulse Shape Discrimination Method Applied to Silicon

Strip Detector

J. Lu, F. Amorini, G. Cardella, A. Di Pietro,

P. Figuera, A. Musumarra, M. Papa, G. Pappalardo,

F. Rizzo and $S$. Tudisco

Section VI. Reaction Mechanisms around the Barrier. Fusion and Fission in Heavy-Ion Reactions

Cross-Sections for Coulomb and Nuclear Breakup of Three-Body 401 Halo Nuclei

E. Garrido, D. V. Fedorov and A. S. Jensen

Multinucleon Transfer Reactions Studied with the PISOLO 405

Spectrometer

L. Corradi, A. M. Stefanini, A. M. Vinodkumar,

S. Beghini, G. Montagnoli, F. Scarlassara and

G. Pollarolo

Study of the Cluster Emission Barrier in ${ }^{12} \mathrm{C}+{ }^{208} \mathrm{~Pb}$ Elastic

Scattering and Possible Observation of Quasimolecular

Configuration

A. A. Ogloblin, K. P. Artemov, Yu. A. Glukhov,

A. S. Dem'yanova, V. V. Paramonov, M. V.Rozhkov,

V. P. Rudakov and S. A. Goncharov

Very Strong Reaction Channels at Barrier Energies in the

System ${ }^{9} \mathrm{Be}+{ }^{209} \mathrm{Bi}$

C. Signorini, A. Andrighetto, J. Y. Guo, L. Stroe,

A. Vitturi, M. Ruan, M. Trotta, F. Soramel, K. E. G. Löbner,

K. Rudolph, I. Thompson, D. Pierroutsakou and M. Romoli

Nuclear Rainbows, Nuclear Matter and the ${ }^{16} \mathrm{O}+{ }^{16} \mathrm{O}$ System

W. von Oertzen, A. Blazevic, H. G. Bohlen, D. T. Khoa,

F. Nouffer, P. Roussel-Chomaz, W. Mittig and

J. M. Cassandjian

Bremsstrahlung by Nonrelativistic Particles in Matter 
Pronounced Airy Structure in Elastic ${ }^{16} \mathrm{O}+{ }^{12} \mathrm{C}$ Scattering at $E_{l a b}=200 \mathrm{MeV}$

Y. A. Gloukhov, A. S. Dem'yanova, A. A. Ogloblin,

M. V. Rozhkov, S. A. Goncharov, R. Julin and W. Trzaska

Fusion Energy Thresholds Predicted with an Adiabatic

Nucleus-Nucleus Potential

J. Wilczyński and K. Siwek-Wilczyńska

Near-Barrier Fusion of ${ }^{36} \mathrm{~S}+{ }^{90,96} \mathrm{Zr}$ : What is the Effect of the Strong Octupole Vibration of ${ }^{96} \mathrm{Zr}$ ?

L. Corradi, A. M. Stefanini, A. M. Vinodkumar,

S. Beghini, G. Montagnoli, F. Scarlassara and M. Bisogno

Dynamical Model of Fission Fragment Angular Distribution

V. A. Drozdov, D. O. Eremenko, O. V. Fotina,

$S$. Yu. Platonov and O. A. Yuminov

Fusion-Fission Reaction ${ }^{12} \mathrm{C},{ }^{16} \mathrm{O}+{ }^{208} \mathrm{~Pb}$ at Subbarrier Energies

S. P. Tretyakova, M. G. Itkis, E. M. Kozulin,

N. A. Kondratiev, I. V. Pokrovskii, E. V. Prokhorova,

L. Calabretta, C. Maiolini, A. Ya. Rusanov and

T. Yu. Tretyakova

Sub-Barrier Fusion with a Halo Nucleus: The ${ }^{6} \mathrm{He}$ Case

M. Trotta, J. l. Sida, N. Alamanos, F. Auger, A. Drouart,

D. J. C. Durand, A. Gillibert, C. Jouanne, V. Lapoux,

A. Lumbroso, F. Marie, S. Ottini, C. Volant,

A. Andreyev, D. L. Balabanski, N. Coulier, G. Georgiev,

M. Huyse, G. Neyens, R. Raabe, S. Ternier,

$P$. van Duppen, K. Vivey, C. Borcea, J. D. Hinnefeld,

A. Musumarra, A. Lèpine and R. Wolski

\section{Concluding Remarks}

Impact of Nuclear Science on Modern Society

R. A. Ricci 
This page is intentionally left blank 


\section{FOREWORD}

The International Nuclear Physics Conference" Bologna 2000. Structure of the Nucleus at the Dawn of the Century", held from May 29 to June 3, 2000 in Bologna, Italy, in the Aula Magna of the University and in the medieval Palazzo dei Notai, was attended by more than 340 scientists from 43 countries and five continents. It was the first time that an International Nuclear Physics Conference of this size had taken place in Bologna.

The Conference was organized by the Physics Department of the University and by the Bologna Section of the Italian National Institute for Nuclear Physics (INFN). It was sponsored, among others, by the Research Directorate General of the European Commission, the University of Bologna, the Italian Physical Society (SIF), the INFN National Laboratories in Legnaro (LNL) and Catania (LNS), the European Centre for Theoretical Studies in Nuclear Physics and Related Areas (ECT*) in Trento, the Italian National Agency for New Technologies, Energy and the Environment (ENEA).

The idea for this Conference emerged from the desire to discuss perspectives in fundamental Nuclear Physics. Some of the most important European research facilities are located in Italy, a country with a long and rich tradition in this field. The Conference took place at a particularly symbolic moment when, celebrating the $900^{t h}$ anniversary of its famous University, Bologna was designated as European City of Culture for the Year 2000. The aim of the Conference was also a cultural contribution to these celebrations and to the commemoration of the $200^{\text {th }}$ anniversary of the death of Luigi Galvani, one of Bologna's most renowned scholars.

In 1997, when Thessaloniki was European City of Culture, the Aristotle University organized a very successful Conference: "Advances in Nuclear Physics and Related Areas". The Bologna event was organized as the second in a hopefully long series of Nuclear Physics Meetings in the European Cities of Culture.

This International Conference Bologna 2000 was devoted to a discipline which has seen a strong revival of research activities in the last decade. New experimental results and theoretical developments in the field of Nuclear Physics will certainly produce important contributions to our knowledge and understanding of Nature's fundamental building blocks.

The interest aroused by the Conference in the scientific community is clearly reflected in the large number of participants. They represented the most important nuclear physics laboratories in the world. The generous grants from our sponsors allowed the financial support for a considerable number of 
participants who, otherwise, would not have been able to attend.

The Conference covered five major topics of modern nuclear physics: nuclear structure, nucleus-nucleus collisions, hadron dynamics, nuclear astrophysics and trans-disciplinary and peaceful applications of nuclear science. The scope of the Conference was to present a review of recent progress in the field and to provide a forum for the discussion of current and future research projects.

The Conference consisted of plenary and parallel sessions. The plenary sessions focused on comprehensive reviews, concepts and perspectives. Specialized talks on recent developments were presented in the parallel and poster sessions. More than 200 participants, among them many young researchers, had the opportunity to present their work in oral contributions.

Two guided tours helped participants and accompanying persons to discover Bologna and the historical roots of its culture and traditions. On the last day most participants joined the excursion to Ravenna and its magnificent Byzantine monuments. We sincerely hope that all the participants enjoyed the Conference and their stay in Bologna.

\section{Acknowledgements}

We are very grateful to the University of Bologna and to its Rector Fabio Roversi Monaco for the generous financial support and the hospitality extended to the Conference in the beautiful Aula Magna of Santa Lucia. We would like to thank the Rector for his warm welcome address. The Pro-rector of the University Ettore Verondini gave an important and encouraging contribution to the organisation of the Conference.

We thank professor Enzo Iarocci, President of the National Institute for Nuclear Physics, for his welcome address.

Our special thanks go to Professor Antonino Zichichi, distinguished senior member of the Department of Physics of the University of Bologna. His promotional activities, his help in the publishing of the Proceedings, and finally his extremely interesting opening lecture, made an essential and animating contribution to the success of the Conference.

The Conference was closed with the most interesting review of the past and the future of Nuclear physics by Professor Renato Angelo Ricci, former President of the Italian Physical Society.

We acknowledge the help of the Department of Physics of the University. We are very grateful to its Director, Professor Attilio Forino, for his numerous suggestions, encouragement and also generous financial help which was essential in the initial stages of the organisation of the Conference. Our spe- 
cial thanks go to the Bologna section of the INFN and its Director Professor Paolo Giusti for the financial and logistic support.

The Research Directorate General of the European Commission, the framework of Human Potential Programme, High-Level Scientific Conferences (under contract no. HPCF-CT-1999-00131), generously supported the participation of young researchers from the European Community and Associate States.

It is a pleasure to thank the President of ENEA, Nobelist Carlo Rubbia, and the head of the ENEA Accelerator-Driven-System Project, Giuseppe Gherardi, for the financial and scientific support offered by ENEA to the Conference.

Essential for the success of the Conference was the dedicated work of the Conference Secretariat: Barbara Simoni and Luisa De Angelis, the Conference Agency: Angela Rizzi, and the kind assistance of Erika Giorgianni. Special thanks go to Angela Belluzzi from the Office of the Rector of the University for her help in organising the Conference venue.

The commitment of ECT* Trento and its Scientific Secretary Renzo Leonardi was invaluable in the organisation and during the Conference. The very efficient work of Ines Campo and Rachel Weatherhead during the five days of the Conference, and the kind assistance of Mauro Meneghini should be particularly mentioned.

We express our warmest thanks to the Directress of the Centro Documentazione delle Donne, Mrs Grazia Negrini, for the kind hospitality extended to the Conference in the magnificent Palazzo dei Notai.

We are very grateful to the municipality of Bologna for hosting the welcome party in the splendid Sala d' Ercole. The visit to the Museo Morandi and to the Town Hall art collections was particularly appreciated.

The financial contribution of the "Fondazione Carisbo" is gratefully acknowledged. Further financial support was provided by the listed in the following pages. Informative brochures were kindly provided by the University of Bologna, by Professor Giorgio Giacomelli, the LNS and LNL and the "Assessorato alla Cultura" of the Bologna Province.

The plenary sessions were recorded and broadcasted in real time via Internet by the INFN informatics and multimedial groups. We express our warmest thanks to the Director Paolo Mazzanti and to Gianfranco Artusi, Daniela Bortolotti, Roberto Giacomelli, Alessandro Italiano, Franco Martelli, Gianluca Peco, Ombretta Pinazza, Giuliano Scandellari, Stefano Zani.

The facilities and services provided by the Physics Department are gratefully acknowledged. We thank Attilio Capponi, Nino Muzzupappa, Romano Serra, Simonetta Salsini and Giuseppe Zaccarelli.

The work of Paolo Finelli was invaluable in preparing the proceedings of 
the Conference.

Our warmest thanks go to the colleagues and friends, members of the Organizing Committee, and to the International Advisory Committee, for the excellent job done in preparing the scientific programme and selecting the speakers. The plenary and parallel sessions were run very smoothly and efficiently by our Session chairpersons.

Finally, we would like to thank all the participants whose enthusiasm, interest and scientific ideas represent the main contribution to the success of the Conference. They presented an exciting variety of new results, demonstrating the vitality of Nuclear Physics and shedding light on future perspectives at the turn of the century.

The Editors 


\title{
WELCOME ADDRESS
}

\author{
FABIO ROVERSI MONACO \\ Rector of the University of Bologna
}

Ladies and Gentlemen, distinguished Colleagues,

It is my pleasure to warmly welcome all the participants in this Conference. The Bologna studium, as you know, is the oldest of the western world: here, the very concept of University as an institution based on independent scientific research and teaching first came into being.

In 1988 the University of Bologna celebrated its nine-hundreth anniversary and, on that occasion, the Magna Charta Universitatum, i.e. the Universal Declaration of Academic rights, was signed by more than 400 Rectors of Universities all over the world. A few months ago, following the same inspiration, an Observatory on Academic Freedom was founded, with the purpose of controlling the respect of the fundamental principles on which scientific research and teaching are based.

This Conference opens at the turn of a millennium in a particularly symbolic moment: the year 2000, when Bologna takes its turn as Cultural Capital of Europe. A century is passing that saw a tremendous progress in understanding the ultimate structure of matter: technological and social consequences have deeply affected the whole mankind in good and evil. Only one hundred years ago Henry Becquerel and Marie Curie discovered radioactivity. Some ten years later, Ernest Rutherford proved the existence, at the core of the atom, of a very small nucleus, where the same kind of energy is stored as the one that feeds our life from the sun. Even the atoms that make up our bodies were synthesized by nuclear reactions in the core of stars and supernova explosions: there is thus an impressive link between macro- and microcosm.

Nevertheless, we assist today to a growing disaffection for science, mainly due to nuclear weapons and improper use of nuclear energy. Let me remind you that the term nuclear was recently dropped from such medical applications as Nuclear Magnetic Resonance. Does this mean that we must give up studying Nature?

The Magna Charta states that the future of mankind depends to a large extent on cultural, scientific and technical development. This means that we must go on investigating the deepest problems Nature faces us with, but, at the same time, we must recover the sense of Nature as a common mother. What we need is a new sense of ethic with a direct commitment of scientists for peace, justice, development and mutual understanding. 
It is the first time a Nuclear Physics Conference of large size takes place in Bologna. In 1977, when Thessaloniki was the Cultural Capital, the Aristotle University organized a very successful Conference " Advances in Nuclear Physics and Related Areas". I hope we are participating in the beginning of a long series of Nuclear Physics meetings to be held in the Cultural Capitals of Europe.

Starting from Enrico Fermi, Italy has a long and rich tradition in this field, and the contribution of Bologna to the advances of nuclear physics covers the main trends in experimental and theoretical fundamental research, carried on mainly by University and INFN, in collaboration with major institutions and laboratories all over the world. It ranges from light nuclei to heavy-ion physics, from nuclear models to hadron structure with a prominent emphasis on high-energy physics and fundamental interactions.

Let me mention now a few words about some troubles that nuclear physics is actually suffering in Bologna. While experimental nuclear physics is represented by many active groups, theoretical nuclear physics runs the risk of disappearing, since young people are moving towards other fields of research where more positions are available. This is a pity in many respects: firstly, a long tradition is disappearing and the richness of knowledge and experience in this field will be definitely lost in a short time; secondly, even if they are considered less fundamental than subnuclear physics, nuclear problems show many interesting and puzzling facets, which may stimulate imagination and development of new models and techniques.

For these reasons, I think that the Physics Department, the Faculty of Sciences and INFN should make a serious effort in term of positions - professors and researchers - to avoid the loss of such a relevant cultural legacy.

The aim of this Conference is to promote the exchange of ideas and future collaboration among nuclear physicists coming from developed countries and developing regions. The high quality of the programme, with special emphasis on new directions and opportunities, the quality of contributions and the level of speakers, who are among the most prominent scientists in this field, the large number of participants, illustrate the vitality of nuclear science and are favourable auspices for the success of this International Conference.

Let me conclude by warmly thanking Giovanni Carlo Bonsignori, Mauro Bruno and the whole organizing committee for their engagement in arranging this meeting. Finally, I wish the Bologna-2000 International Conference on Structure of the Nucleus at the Dawn of the Century the best success, and I hope you will enjoy not only the conference, but also your stay in Bologna and its University. 


\section{Conference Organization}

CONFERENCE CHAIRMEN:

G. Bonsignori (Bologna), M. Bruno (Bologna)

SCIENTIFIC SECRETARIES:
A. Ventura (ENEA/Bologna),
D. Vretenar (Zagreb)

\section{INTERNATIONAL ADVISORY COMMITTEE}

M. Arnould (Bruxelles)

J. Aysto (Jyvaskyla)

C. Baktash (Oak Ridge)

D. Bazzacco (Padova)

J.-F. Berger (CEA/Bruyères-le-Châtel)

J.-P. Blaizot (CEA/Saclay)

S. Boffi (Pavia)

J. Bondorf (Copenhagen)

R. Bougault (Caen)

T. Bressani (Torino)

R. Broglia (Milano)

P. Chomaz (Caen)

C. Ciofi degli Atti (Perugia)

M. Di Toro (Catania)

C. Fahlander (Lund)

G. Fiorentini (Ferrara)

B. Frois (CEN Saclay)

K. Gelbke (NSCL/East Lansing)

W. Gelletly (Surrey)

G. Gherardi (ENEA/Bologna)

\section{LOCAL ORGANIZING COMMITTEE}

F. Cannata (Bologna)

A. Covello (Napoli)

G. De Angelis (Legnaro)

P. Finocchiaro (Catania)

R. Leonardi (Trento)

G. Lo Bianco (Camerino)
D.H.E. Gross (Berlin)

M. Grypeos (Thessaloniki)

W. Haxton (Seattle)

F. Iachello (New Haven)

A.V. Ignatyuk(Obninsk)

M. Ishihara (Tokyo)

F. Kaeppeler (Karlsruhe)

P. Kienle (Munchen)

R. Malfliet (Trento)

I. Massa (Bologna)

V.Metag (Giessen)

E. Migneco (Catania)

Y.Ts. Oganessian (Dubna)

T. Otsuka (Tokyo)

R.A. Ricci (Padova)

P. Ring (Munchen)

F.-K. Thielemann (Basel)

A.W. Thomas (Adelaide)

A. Zichichi (Bologna)

S. Lunardi (Padova)

A. Mengoni (ENEA/Bologna)

A. Pagano (Catania)

M. Savoia (Bologna)

A. Vitturi (Padova) 


\section{SPONSORS}

- Dipartimento di Fisica dell'Universita' di Bologna

- Istituto Nazionale di Fisica Nucleare - Sezione di Bologna

- Universita' degli Studi di Bologna

- Provincia di Bologna

- INFN - Laboratori Nazionali del Sud - Catania

- INFN - Laboratori Nazionali di Legnaro - Legnaro PD

- ENEA

- Research Directorate General of the European Commission (Under contract no. HPCF-CT-1999-00131)

- ECT* - European Centre for Theoretical Studies in Nuclear Physics and Related Areas

- Societa' Italiana di Fisica

- Fondazione Carisbo, Bologna

- Banca di Roma, Roma

- Bologna 2000 Committee

- EG\&G ORTEC Italia

- CAEN - Nuclear Physics - Viareggio LU

- 3M Italia

- Consorzio Marchio Storico dei Lambruschi Modenesi

- Consorzio tutela Parmigiano Reggiano

- Consorzio produttori di aceto balsamico tradizionale di Modena

- Alcisa Bologna

- Consorzio tutela aceto balsamico

- Monari e Federzoni - Bomporto (Modena) 


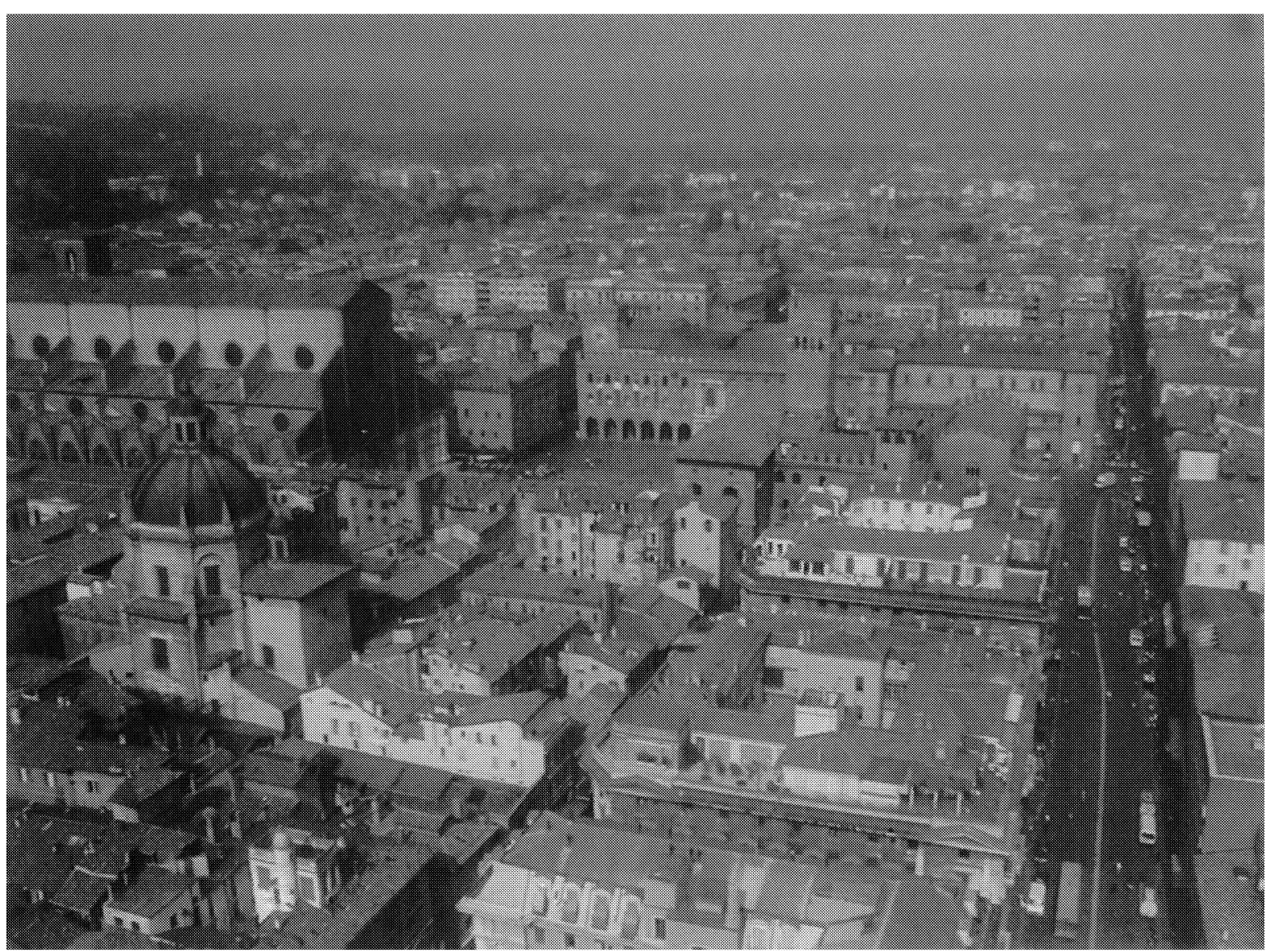




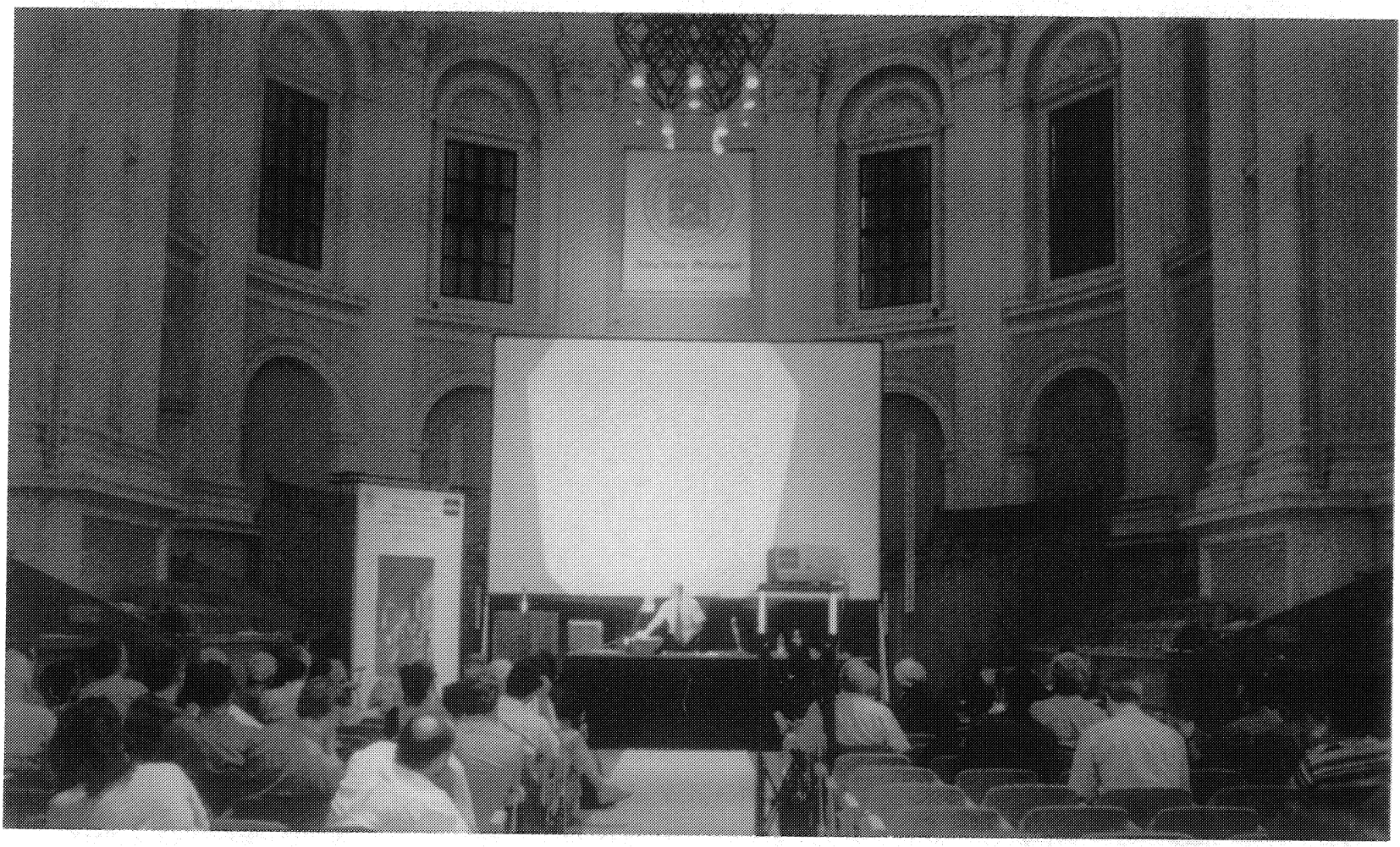




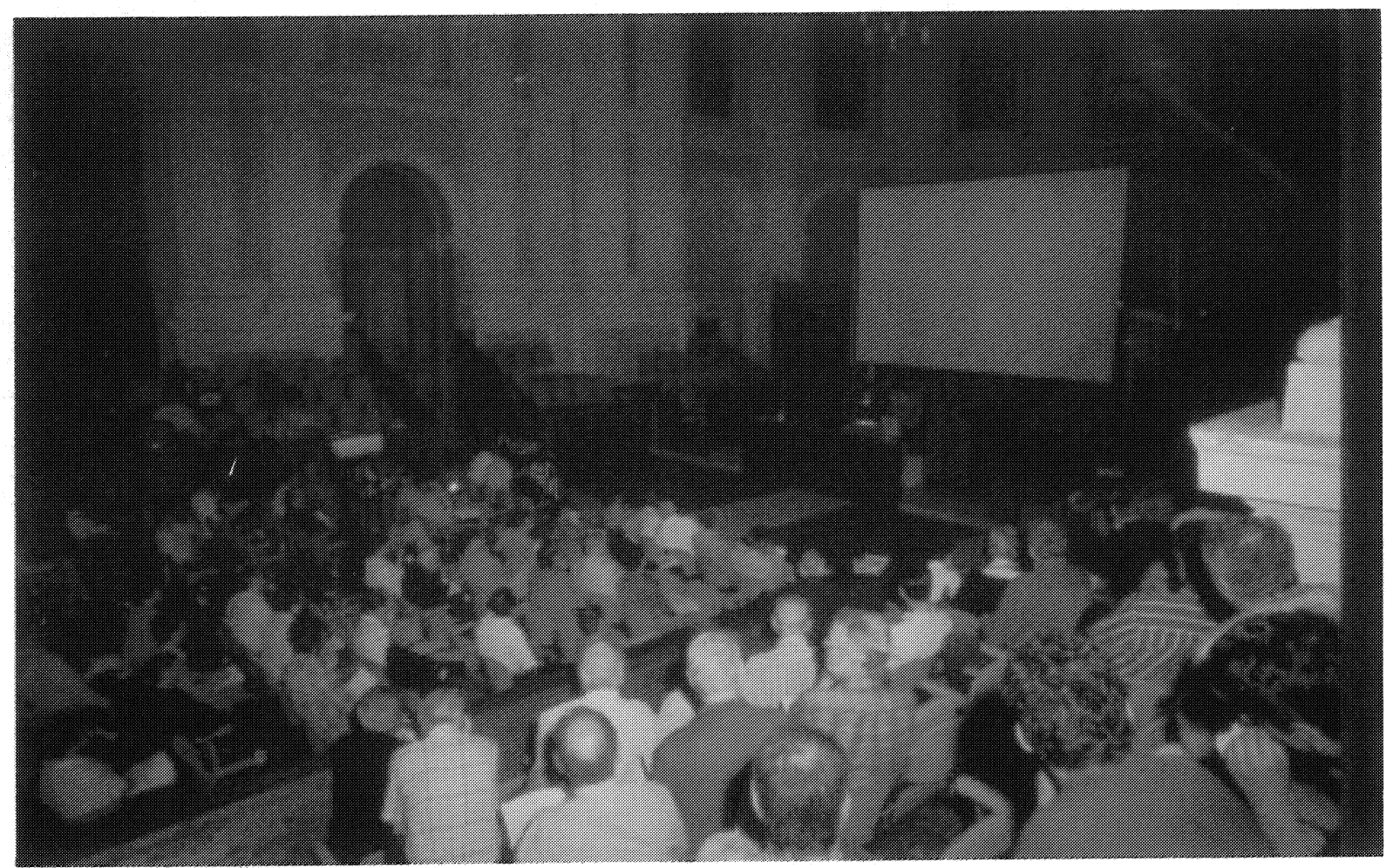




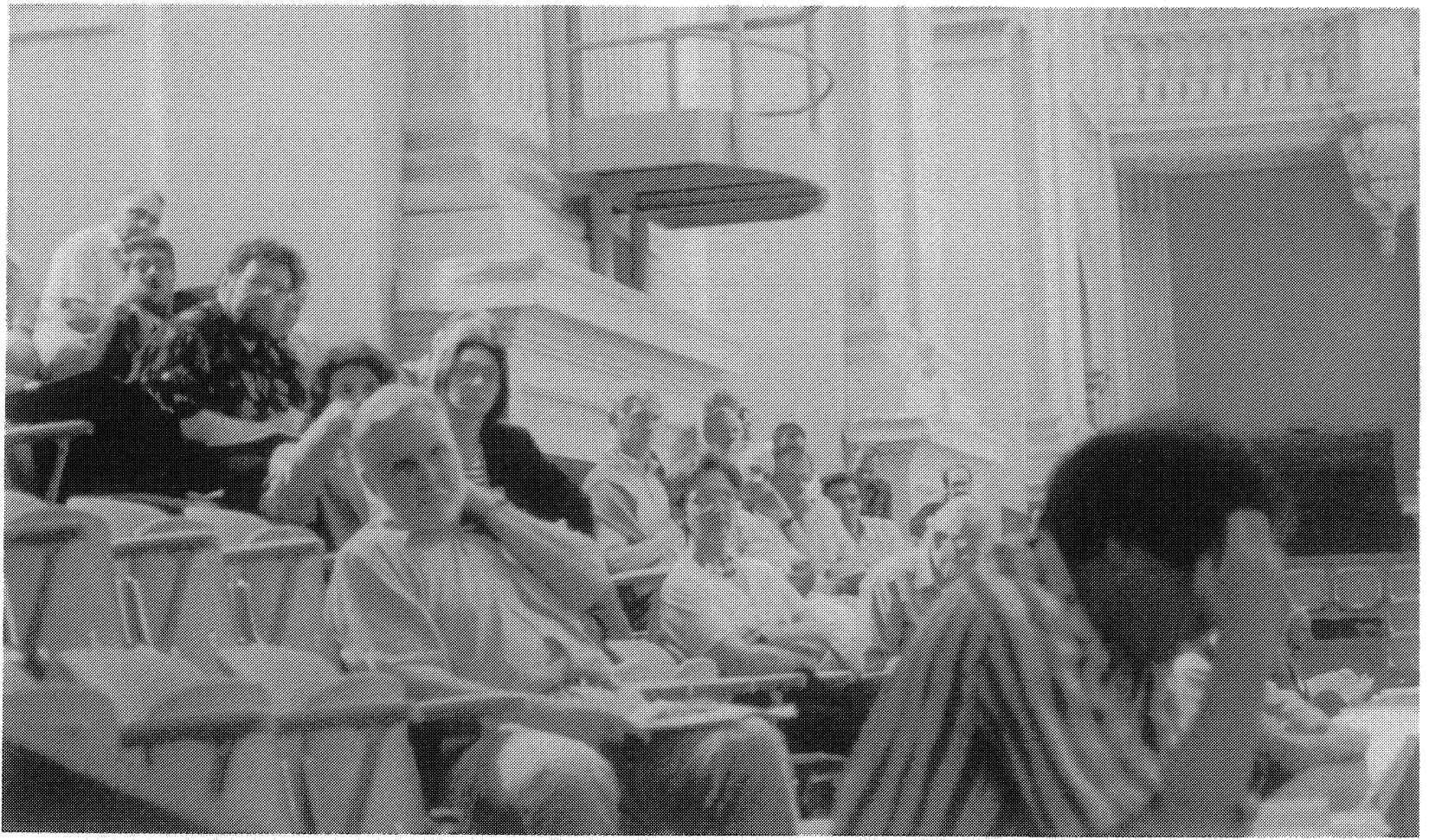




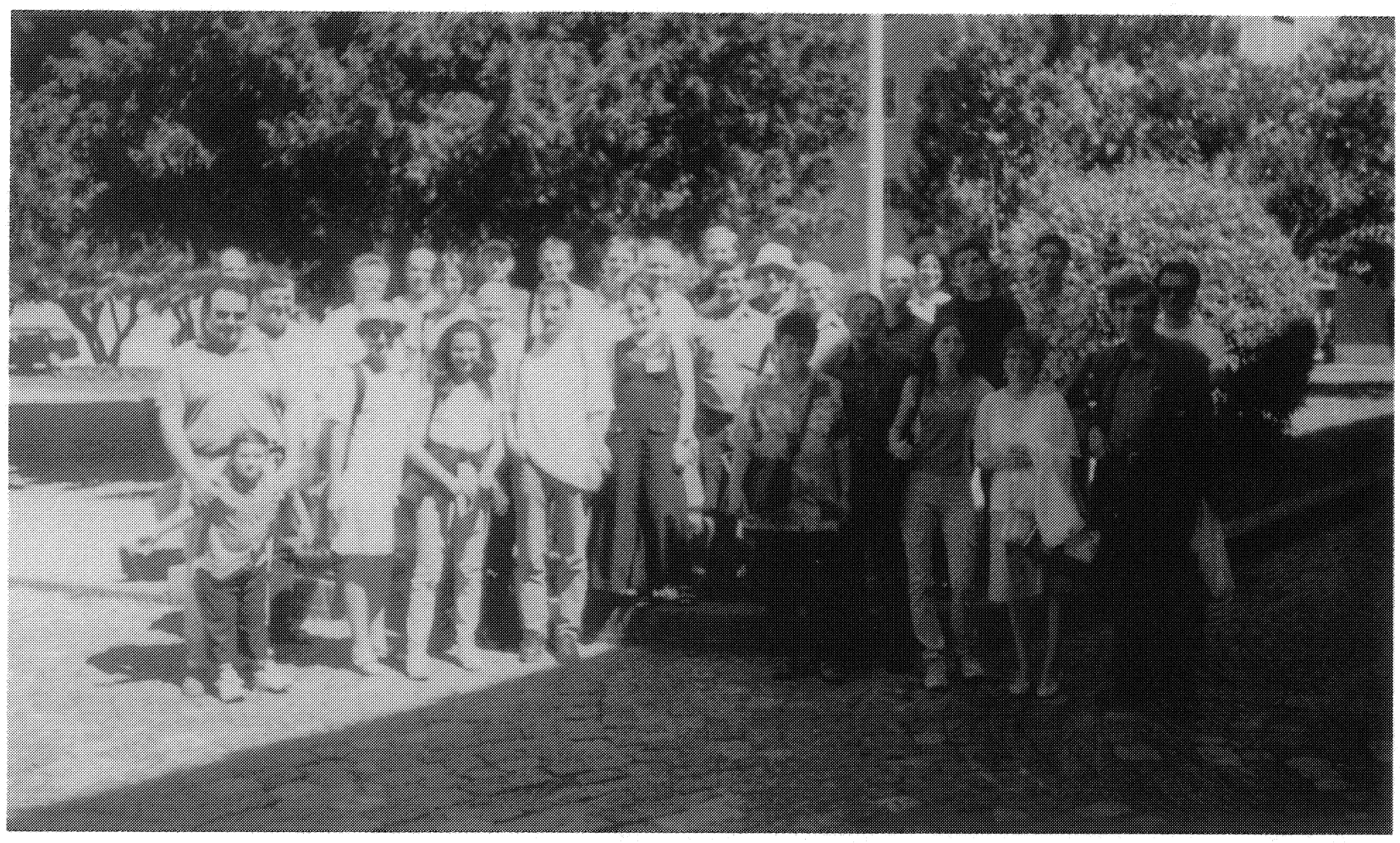

